\title{
Florística dos componentes arbóreo e arbustivo de um trecho da Floresta Estacional Semidecídua Montana, município de Pedreira, estado de São Paulo
}

\author{
LEILA F. YAMAMOTO ${ }^{1}$, LUIZA S. KINOSHITA ${ }^{2,3}$ e FERNANDO R. MARTINS ${ }^{2}$
}

(recebido: 28 de novembro de 2001; aceito: 25 de novembro de 2004)

\begin{abstract}
Floristic analysis of trees and shrubs in a fragment of Semideciduous Montane Forest, municipality of Pedreira, São Paulo State, southeastern Brazil). The aims of this study were to know the species of trees and shrubs in a fragment of Semideciduous Montane Forest and investigate the floristic difference between Montane and Submontane forests in São Paulo State. To survey tree and shrub species, weekly collectings were done during 15 months in the Fazenda Bela Vista (46 ${ }^{\circ} 52^{\prime} \mathrm{W}$ and $22^{\circ} 47^{\prime} \mathrm{S}, 750-850 \mathrm{~m}$ ). A total of 151 species of 106 genera and 47 angiosperm families was collected. Leguminosae, Myrtaceae, Lauraceae, Rubiaceae, Meliaceae, Piperaceae, Solanaceae, Ocotea, Piper, Machaerium, Miconia, Eugenia and Solanum were the richest taxa. Samples performed by other authors of the Montane and Submontane formations of the Seasonal Semideciduous Forest were compared. Leguminosae, Euphorbiaceae, Myrtaceae, Rubiaceae, Lauraceae, Machaerium, Eugenia, Solanum and Croton had higher relative constancy and richness of tree and shrub species in both formations. The $\mathrm{G}$ test indicated that Solanaceae, Asteraceae, Melastomataceae, Aspidosperma, Trichilia and Casearia had significantly higher species richness and relative constancy in the Montane formation. On the other hand, Meliaceae, Rutaceae, Moraceae, Ocotea, Miconia, Myrcia and Ficus had significantly higher richness and constancy in the Submontane formation. Therefore, each formation has distinct floristic composition at the species as well as at genera and family levels.
\end{abstract}

Key words - floristics, floristic comparison, São Paulo state, Semideciduous Montane Forest, Semideciduous Submontane Forest

RESUMO - (Florística dos componentes arbóreo e arbustivo de um trecho da Floresta Estacional Semidecídua Montana, município de Pedreira, estado de São Paulo). Este estudo teve como objetivos conhecer a flora de árvores e arbustos em um fragmento da Floresta Estacional Semidecídua Montana e verificar se há diferença entre as formações Montana e Submontana no estado de São Paulo. Durante 15 meses foram feitas coletas semanais de flores e/ou frutos de espécies de arbustos, arvoretas, árvores e palmeiras, através de caminhadas nos fragmentos na Fazenda Bela Vista ( $4^{\circ} 52^{\prime} \mathrm{W}$ e $22^{\circ} 47^{\prime} \mathrm{S}, 750$ a $850 \mathrm{~m}$ de altitude). Foram identificadas 151 espécies de 106 gêneros e 47 famílias de angiospermas, sendo os táxons mais ricos em espécies Leguminosae, Myrtaceae, Lauraceae, Rubiaceae, Meliaceae, Piperaceae e Solanaceae, Ocotea, Piper, Machaerium, Miconia, Eugenia e Solanum. Foram comparados levantamentos das formações Montana e Submontana da Floresta Estacional Semidecídua no estado de São Paulo. Os táxons de maior constância relativa e maior riqueza de espécies arbustivas e arbóreas em ambas as formações foram: Leguminosae, Euphorbiaceae, Myrtaceae, Rubiaceae, Lauraceae, Machaerium, Eugenia, Solanum e Croton. A aplicação do teste G indicou que Solanaceae, Asteraceae, Melastomataceae, Aspidosperma, Trichilia e Casearia apresentaram riqueza específica e constância relativa significativamente maiores na formação Montana que na Submontana. Por outro lado, Meliaceae, Rutaceae, Moraceae, Ocotea, Miconia, Myrcia e Ficus apresentaram riqueza e constância significativamente maiores na formação Submontana. Portanto, na Floresta Estacional Semidecídua no estado de São Paulo, há distinção florística entre as formações Montana e Submontana tanto em nível de espécies quanto de gênero e família.

Palavras-chave - florística, comparação florística, estado de São Paulo, Floresta Estacional Semidecídua Montana, Floresta Estacional Semidecídua Submontana

\section{Introdução}

A vegetação da Floresta Estacional Semidecídua ocorrente no Brasil acima de $500 \mathrm{~m}$ de altitude e entre

1. Universidade Estadual de Campinas, Instituto de Biologia, Curso de Pós-Graduação em Biologia Vegetal, Caixa postal 6109, 13083-970, Campinas, SP, Brasil

2. Universidade Estadual de Campinas, Instituto de Biologia, Departamento de Botânica, Caixa postal 6109, 13083-970 Campinas, SP, Brasil

3._Autor para correspondência: luizakin@unicamp.br $16^{\circ}$ e $24^{\circ}$ de latitude sul foi classificada na formação Montana (Veloso et al. 1991). No entanto, há discordância quanto à altitude mínima de seu limite com a formação Submontana. Meira Neto et al. (1989) e Leitão Filho (1992) consideraram $1.000 \mathrm{~m}$ como a altitude a partir da qual ocorreria uma mudança conspícua na composição florística e na estrutura das florestas paulistas. Salis et al. (1995) encontraram dois grupos florísticos distintos de florestas no estado de São Paulo: um acima e outro abaixo de $700 \mathrm{~m}$. Torres et al. (1997) encontraram uma altitude mediana entre 700 e 
$750 \mathrm{~m}$ separando a flora das florestas estacionais baixoaltitudinais e alti-altitudinais paulistas. Assim, no estado de São Paulo, o limite entre a Floresta Estacional Semidecídua Montana (FESM) e a Floresta Estacional Semidecídua Submontana (FESS) estaria entre 500 e $1.000 \mathrm{~m}$ de altitude, com maior freqüência ao redor de 700 a $750 \mathrm{~m}$. A transição entre essas formações deve ocorrer de forma gradual, sendo os limites entre elas estabelecidas de modo artificial (Oliveira Filho et al. 1994).

A FESM diferiria das formações de altitudes mais baixas tanto na flora quanto na estrutura (Leitão Filho, 1992). Sua flora seria constituída por algumas espécies exclusivas e outras que aí são mais abundantes, mas também ocorreriam em outras formações florestais (Meira Neto et al. 1989). A FESM apresenta uma fisionomia marcada por árvores baixas com altura média em torno de 7 a $8 \mathrm{~m}$, podendo ocorrer poucos indivíduos emergentes; o estrato arbóreo é denso, com as árvores muito próximas umas das outras, apresentando copas sobrepostas, que acarretam um forte sombreamento do solo (Leitão Filho 1992). A maioria das árvores não tem tronco com diâmetro (à altura do peito) muito grande, geralmente menor que $15 \mathrm{~cm}$ e os estratos arbustivo e herbáceo apresentam-se pouco desenvolvidos, quando comparados com os de florestas em altitudes mais baixas (Rodrigues et al. 1989). Geralmente, os solos da FESM são ácidos, erodidos, rasos e mais pobres em nutrientes (Leitão Filho 1992, Torres et al. 1997).

$\mathrm{O}$ artigo $2^{\circ}$ do Código Florestal (lei número 4771 de 15/09/1965) diz que florestas em topos de morro ou em vertentes muito inclinadas constituem área de preservação permanente. Geralmente, a FESM ocorre em topos de morro ou em vertentes, situação que aumenta sua importância como elemento protetor do relevo contra a erosão e como conservador da biodiversidade. Os fragmentos florestais do estado de São Paulo apresentam diferentes estádios sucessionais em decorrência de diferentes tipos e intensidades de perturbação antrópica. É importante estudar áreas nessas condições, para poder conhecer melhor a flora constituinte de florestas perturbadas e indicar espécies apropriadas à revegetação de áreas devastadas.

Torres et al. (1997) encontraram uma similaridade específica muito baixa entre os levantamentos da Floresta Estacional Semidecídua no estado de São Paulo e concluíram que os baixos valores de similaridade indicariam um padrão predominante de distribuição geográfica restrita, em que as espécies arbóreas tenderiam a ocorrer apenas em um ou poucos locais. Torres et al. (1997) também chamaram atenção para o fato de que a diversidade alfa da Floresta Estacional Semidecídua paulista, estimada pelo índice H'de Shannon, tem a mesma ordem de grandeza que a de florestas amazônicas de terra firme. Isso significa que a pequena área de floresta natural representada pelos fragmentos ainda existentes no interior do estado de São Paulo preserva uma diversidade muito alta e pouco conhecida.

Considerando que a Floresta Estacional Semidecídua Montana tem grande importância ecológica e ambiental e que os estudos feitos até o presente têm mostrado que suas espécies são diferentes das ocorrentes na Floresta Estacional Semidecídua Submontana, este estudo tem como objetivos: 1) conhecer a composição florística de um fragmento da Floresta Estacional Semidecídua Montana, apresentando uma lista das espécies arbóreas e arbustivas que aí ocorrem; e 2) verificar se também há diferenças na composição das famílias e gêneros mais ricos em espécies arbóreas entre as formações Montana e Submontana da Floresta Estacional Semidecídua do estado de São Paulo.

\section{Material e métodos}

A floresta estudada localiza-se no município de Pedreira, estado de São Paulo, na região geomorfológica do Planalto Atlântico, na zona da Morraria de Lindóia, que apresenta relevo montanhoso com serras alongadas de topos angulosos e vertentes ravinadas com perfis retilíneos e, às vezes, abruptos (Ponçano et al. 1981). O clima é tropical com chuvas de verão (figura 1), classificado como tipo II de Walter \& Lieth (Walter 1972). O solo predominante na região de Pedreira é o Argissolo Vermelho-Amarelo, correspondente, na classificação antiga, a Podzólico Vermelho-Amarelo (Oliveira et al. 1999).

Kuhlmann (1942), Kuhlmann \& Kühn (1947) e Toledo Filho et al. (1993) estudaram trechos da FESM na Morraria de Lindóia, onde a vegetação é pouco conhecida e com poucas coletas botânicas. No município de Pedreira, os fragmentos florestais remanescentes têm, geralmente, área pequena e são fortemente perturbados. A área de estudo localiza-se na Fazenda Bela Vista, uma propriedade particular no bairro de Entre-Montes, nas coordenadas $46^{\circ} 52^{\prime} \mathrm{W}$ e $22^{\circ} 47^{\prime} \mathrm{S}$, em altitudes (medidas com altímetro aneróide no local de estudo) entre 750 e $850 \mathrm{~m}$. A mata está fragmentada e tem no total cerca de 100 ha, estendendo-se pelas fazendas vizinhas. Apresenta sinais de perturbação, com presença de cepas indicando corte de árvores, muitas descontinuidades no dossel e sem distinção entre os estratos. Em alguns locais, há resquícios de queimada recente (troncos com fuligem); em outros, desde 1984 não ocorrem queimadas (segundo informações dos moradores locais). Na borda, há grande 


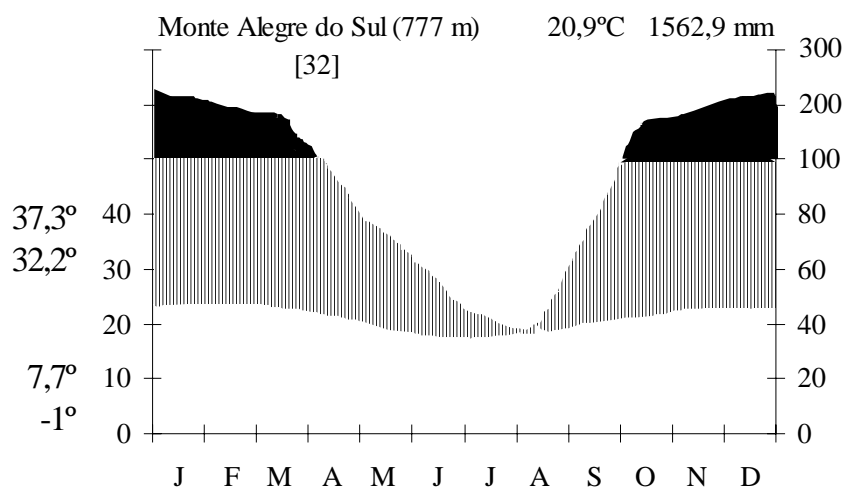

Figura 1. Diagrama climático de Walter \& Lieth do clima tipo II (tropical com chuvas de verão) da região do município de Pedreira, estado de São Paulo. Fonte: Centro Integrado de Informação Agrometeorológicas/Instituto Agronômico de Campinas.

Figure 1. Walter \& Lieth's climatic diagram type II (tropical with Summer rain climate) of the municipality of Pedreira region, São Paulo State. Data source: Centro Integrado de Informação Agrometeorológicas/Instituto Agronômico de Campinas.

quantidade de lianas e, no interior da mata, há poucos epífitos. A floresta faz divisa com pastagens e uma plantação de eucalipto.

A floresta estudada foi escolhida pelas facilidades logísticas oferecidas pelo proprietário, pelas facilidades de acesso, por representar um remanescente florestal razoável tanto pela sua extensão quanto pelo seu estado de conservação, quando comparado com outros remanescentes da região, e por haver poucos trabalhos realizados em florestas de altitude da região.

As coletas foram feitas semanalmente de agosto de 1997 a meados de outubro de 1998, através de caminhadas assistemáticas pelos fragmentos. Foram coletados indivíduos com DAP (diâmetro do tronco à altura do peito) igual ou superior a $3 \mathrm{~cm}$, cuja forma de crescimento fosse árvore, arvoreta, arbusto ou palmeira. Consideraram-se como arbustos os indivíduos lenhosos ou semilenhosos (indivíduos herbáceos com a base do caule lenhosa) com ramificações permanentes originando-se do caule até $50 \mathrm{~cm}$ acima do solo. Indivíduos lenhosos com ramificações acima daquela medida foram considerados arvoretas (até $4 \mathrm{~m}$ de altura) ou árvores. Os indivíduos amostrados receberam uma plaqueta de alumínio numerada, para auxiliar no controle do levantamento. As flores e/ou frutos mais delicados foram preservados numa solução aquosa de etanol 70\%. Fizeram-se exsicatas de todas as espécies coletadas, depositando-se o material-testemunho no Herbário do Departamento de Botânica da Universidade Estadual de Campinas (UEC).

Adotou-se o sistema de Cronquist (1981, 1988), mas as leguminosas foram consideradas uma só família (Polhill \&
Raven 1981). A identificação foi feita através da literatura e por comparação com as exsicatas do herbário UEC. Foram também consultados os herbários SPF (Departamento de Botânica da Universidade de São Paulo), SPSF (Instituto Florestal), SP (Instituto de Botânica) e IAC (Instituto Agronômico de Campinas). Foram consultados especialistas para identificar alguns materiais ou para confirmar as identificações. Os nomes dos autores das espécies foram abreviados conforme Brummitt \& Powell (1992). Os nomes populares foram obtidos dos auxiliares de campo, dos moradores locais e de diversas fontes bibliográficas.

$\mathrm{Na}$ comparação entre as formações Montana e Submontana da Floresta Estacional Semidecídua no estado de São Paulo, consideraram-se as oito famílias e gêneros com maior constância relativa e mais ricos em espécies arbóreas e arbustivas nos diversos levantamentos estudados. Os oito gêneros e famílias analisados abrangem uma grande proporção do total de espécies arbóreas e arbustivas presentes em cada levantamento (tabela 1). Sendo assim, considerar apenas os oito gêneros e famílias de maior riqueza específica simplifica as análises, torna-as mais objetivas e, ao mesmo tempo, inclui uma parte muito significativa do total de espécies de cada local, diminuindo o ruído que seria incluído nas análises, se todos os táxons fossem considerados (Gauch 1982). A constância relativa de um táxon $\mathrm{CR}_{\mathrm{t}}$ considera o número de levantamentos com presença do táxon $\mathrm{P}_{\mathrm{t}}$ em relação ao total de levantamentos T (MüellerDombois \& Ellenberg 1974): $\mathrm{CR}_{\mathrm{t}}=100 \mathrm{P} / \mathrm{T}$. A significância da diferença da constância relativa de cada família ou gênero entre a FESM e a FESS foi verificada através do teste G (Sokal \& Rohlf 1995). Nessa comparação, foram considerados 15 levantamentos na FESS (Bertoni 1984, Cavassan et al. 1984, Pagano \& Leitão Filho 1987, Bertoni et al. 1988, Matthes 1980, Catharino 1989, Vieira et al. 1989, Cesar \& Leitão Filho 1990, Nicolini-Gabriel \& Pagano 1992, Gabriel \& Pagano 1993, Costa \& Mantovani 1995, Bernacci \& Leitão Filho 1996, Rozza 1997, Santos \& Kinoshita 2003, Stranghetti \& Ranga 1998). Na FESM do estado de São Paulo, foram considerados 16 levantamentos (Kuhlmann \& Kühn 1947, Baitello \& Aguiar 1982, Mattos \& Mattos 1982, Meira Neto et al. 1989, Rodrigues et al. 1989, Silva 1989, Torres 1989, Robim et al. 1990, Baitello et al. 1992, Pastore et al. 1992, Toledo Filho et al. 1993; Kotchetkoff-Henriques \& Joly 1994, Rossi 1994, CardosoLeite 1995, Gandolfi et al. 1995), além do presente estudo.

\section{Resultados}

Foram identificadas 151 espécies em 106 gêneros e 47 famílias (tabela 2). Dois espécimes foram identificados apenas até o nível de gênero. Das espécies amostradas, 97 foram árvores, duas palmeiras, 24 arbustos e 28 arvoretas.

As famílias com maior número de espécies na mata da Fazenda Bela Vista foram: Leguminosae (31 
Tabela 1. Proporção dos oito gêneros mais ricos em espécies em relação ao total de gêneros (\% gêneros) e das oito famílias mais ricas em espécies em relação ao total de famílias (\% famílias) em 31 levantamentos de florestas estacionais semidecíduas no estado de São Paulo. Entre parênteses, a relação do número de espécies pertencentes aos oito gêneros ou oito famílias pelo número total de espécies em cada localidade.

Table 1. Ratio of the eight richest genera or families to the total number of genera or families in 31 surveys of seasonal semideciduous forests in São Paulo State. Between brackets, the ratio of the number of species belonging to these genera or families to the total number of species in each survey.

\begin{tabular}{|c|c|c|c|}
\hline Locais & \% Gêneros & \% Famílias & Autores \\
\hline Águas da Prata & $7,7(16,7)$ & $17,8(46,0)$ & Toledo et al. 1993 \\
\hline Amparo & $4,1(18,1)$ & $13,1(54,3)$ & Kuhlmann \& Kühn 1947 \\
\hline Anhembi & $7,6(20,9)$ & $16,7(41,7)$ & Cesar \& Leitão Filho 1990 \\
\hline Angatuba & $6,1(19,0)$ & $13,3(45,1)$ & Torres 1989 \\
\hline Atibaia & $5,3(23,2)$ & $14,3(47,2)$ & Meira Neto et al. 1989 \\
\hline Bauru & $4,0(18,3)$ & $25,0(51,7)$ & Cavassan et al. 1984 \\
\hline Botucatu & $7,5(22,1)$ & $16,7(51,4)$ & Gabriel \& Pagano 1993 \\
\hline Campinas (B. Jequitibás) & $8,1(29,8)$ & $19,0(74,2)$ & Matthes 1980 \\
\hline Campinas (R. Cachoeira) & $6,7(20,6)$ & $16,3(54,3)$ & Santos \& Kinoshita 2003 \\
\hline Campinas (S. Vicente) & $7,8(18,2)$ & $17,8(57,7)$ & Bernacci \& Leitão Filho 1996 \\
\hline Campos do Jordão & $21,6(42,8)$ & $42,1(80,3)$ & Mattos \& Mattos 1982 \\
\hline Campos do Jordão & $6,1(26,6)$ & $13,6(61,4)$ & Robim et al. 1990 \\
\hline Cantareira & $7,3(28,0)$ & $16,7(58,2)$ & Baitello \& Aguiar 1982 \\
\hline Cantareira (Pinheirinho) & $8,6(28,4)$ & $18,2(56,0)$ & Baitello et al. 1992 \\
\hline Guarulhos & $7,5(22,1)$ & $17,0(60,5)$ & Gandolfi et al. 1995 \\
\hline Itirapina & $12,7(28,2)$ & $25,0(57,6)$ & Kotchetkoff-Henriques \& Joly 1994 \\
\hline Jahu & $6,7(18,9)$ & $17,8(52,1)$ & Nicolini-Gabriel \& Pagano 1992 \\
\hline Jundiaí (Serra do Japi) & $8,8(26,6)$ & $17,4(55,7)$ & Rodrigues et al. 1989 \\
\hline Matão & $6,2(22,7)$ & $14,8(55,7)$ & Rozza 1997 \\
\hline Paulo Faria & $9,3(20,5)$ & $20,5(47,3)$ & Stranghetti \& Ranga 1998 \\
\hline Pedreira & $7,5(21,2)$ & $17,0(52,3)$ & Presente trabalho \\
\hline Piracicaba & $6,3(19,9)$ & $19,5(59,2)$ & Catharino 1989 \\
\hline Piracicaba (Ibicatu) & $10,0(21,3)$ & $20,5(56,3)$ & Costa \& Mantovani 1995 \\
\hline Porto Ferreira & $7,1(19,3)$ & $17,8(58,1)$ & Bertoni 1984 \\
\hline Rio Claro & $6,1(17,4)$ & $15,1(49,7)$ & Pagano \& Leitão Filho 1987 \\
\hline Santa Rita Passa Quatro (Capetinga Oeste) & $11,6(17,3)$ & $21,6(56,8)$ & Vieira et al. 1989 \\
\hline Santa Rita do Passa Quatro (Praxedes) & $14,0(20,5)$ & $28,6(61,6)$ & Bertoni et al. 1988 \\
\hline São Bernardo dos Campos & $14,5(24,0)$ & $24,2(54,7)$ & Pastore et al. 1992 \\
\hline São José dos Campos & $6,8(26,1)$ & $15,4(54,4)$ & Silva 1989 \\
\hline São Paulo (Cidade Universitária) & $9,6(29,4)$ & $23,5(62,2)$ & Rossi 1994 \\
\hline São Roque & $6,3(21,1)$ & $14,8(54,1)$ & Cardoso-Leite 1995 \\
\hline
\end{tabular}

espécies, das quais 15 Papilionoideae, 9 Caesalpinioideae e 7 Mimosoideae), Myrtaceae (11), Lauraceae e Rubiaceae (7), Meliaceae, Piperaceae e Solanaceae (6), Asteraceae, Euphorbiaceae e Rutaceae (5). Essas dez famílias juntas apresentaram 89 espécies, constituindo $58,9 \%$ das espécies amostradas, das quais Leguminosae representou $20,53 \%$. As 62 espécies restantes distribuíram-se em 37 famílias.

Os gêneros com maior riqueza de espécies foram Ocotea e Piper (cinco espécies); Eugenia, Machaerium, Miconia e Solanum (quatro espécies), e Acacia, Cordia, Dalbergia, Ficus, Lonchocarpus, Myrsine, Tabebuia, Trichilia e Zanthoxylum com três espécies cada um. Sete gêneros contribuíram com duas espécies e 84 gêneros com apenas uma espécie.

As famílias de maior riqueza específica na FESM no estado de São Paulo apresentaram valores de constância relativa de $50 \%$ ou mais (tabela 3 ): Euphorbiaceae, Leguminosae, Myrtaceae, Rubiaceae, Lauraceae, Solanaceae, Asteraceae e Melastomataceae, tendo Euphorbiaceae uma constância relativa de $100 \%$. Na FESS, as famílias de maior riqueza 
Tabela 2. Espécies da Floresta Estacional Semidecídua Montana da Fazenda Bela Vista, Pedreira, SP. Coletora L.F. Yamamoto. $\mathrm{P}=$ porte, $\mathrm{av}=$ árvore, $\mathrm{at}=$ arvoreta, $\mathrm{ab}=$ arbusto, $\mathrm{pa}=$ palmeira.

Table 2. Species of the Montane Semideciduous Seasonal Forest in the Bela Vista farm, municipality of Pedreira, São Paulo State. Colletor: L.F. Yamamoto. $\mathrm{P}=$ habit, $\mathrm{av}=$ tree, $\mathrm{at}=$ treelet, $\mathrm{ab}=$ shrub, $\mathrm{pa}=$ palm.

\begin{tabular}{|c|c|c|c|}
\hline Família/Espécie & Nome popular & $\mathrm{P}$ & $\mathrm{N}^{\circ}$ de coleta \\
\hline \multicolumn{4}{|l|}{ ACANTHACEAE } \\
\hline Justicia sp. & & $\mathrm{ab}$ & 161 \\
\hline \multicolumn{4}{|l|}{ ANACARDIACEAE } \\
\hline Tapirira obtusa (Benth.) Mitchell & peito-de-pomba & av & 33,82 \\
\hline \multicolumn{4}{|l|}{ ANNONACEAE } \\
\hline Annona cacans Warm. & araticum & av & 147,166 \\
\hline Rollinia silvatica (A.St.-Hil.) Mart. & araticum & av & 109,268 \\
\hline \multicolumn{4}{|l|}{ APOCYNACEAE } \\
\hline Aspidosperma camporum Müll.Arg. & & av & 21,278 \\
\hline \multicolumn{4}{|l|}{ AQUIFOLIACEAE } \\
\hline Ilex cerasifolia Reissek & & av & 84 \\
\hline \multicolumn{4}{|l|}{ ARALIACEAE } \\
\hline Dendropanax cuneatum (DC.) Decne. \& Planch. & maria-preta & av & 221 \\
\hline \multicolumn{4}{|l|}{ ARECACEAE } \\
\hline Bactris setosa Mart. & tucum-bravo & pa & 290 \\
\hline Syagrus romanzoffiana (Cham.) Glassm. & jerivá & pa & 252 \\
\hline \multicolumn{4}{|l|}{ ASTERACEAE } \\
\hline Eupatorium inulaefolium Kunth & & at & 203 \\
\hline Gochnatia polymorpha (Less.) Cabrera & cambará & av & $18,48,90$ \\
\hline Piptocarpha macropoda (DC.) Baker & vassoura-preta & av & 248 \\
\hline Senecio brasiliensis (Spreng.) Less. & & at & 259 \\
\hline Vernonia polyanthes Less. & assa-peixe & at & 253 \\
\hline \multicolumn{4}{|l|}{ BIGNONIACEAE } \\
\hline Tabebuia chrysotricha (Mart. ex DC.) Standl. & ipê-cascudo & at & 250 \\
\hline Tabebuia ochracea (Cham.) Standl. & ipê-do-campo & av & 249 \\
\hline Tabebuia serratifolia (Vahl.) Nichols & ipê-amarelo & av & 20 \\
\hline Zeyheria tuberculosa (Vell.) Bureau & bolsa-de-pastor & av & 235 \\
\hline \multicolumn{4}{|l|}{ BOMBACACEAE } \\
\hline Chorisisa speciosa A.St.-Hil. & paineira-rosa & av & 117 \\
\hline Eriotheca candolleana (K.Schum.) A.Robyns & embiruçu & av & 45 \\
\hline Pseudobombax grandiflorum (Cav.) A.Robyns & paina-branca & av & 224 \\
\hline \multicolumn{4}{|l|}{ BORAGINACEAE } \\
\hline Cordia ecalyculata Vell. & café-de-bugre & av & 86 \\
\hline Cordia sellowiana Cham. & capitão-do-mato & av & $7,206,6$ \\
\hline Cordia trichotoma (Vell.) Arrab. ex Steud. & louro-pardo & av & 19,209 \\
\hline Patagonula americana $\mathrm{L}$. & guaiuvira & av & 87 \\
\hline \multicolumn{4}{|l|}{ CECROPIACEAE } \\
\hline Cecropia glaziovi Snethl. & embaúba & av & 239 \\
\hline \multicolumn{4}{|l|}{ CELASTRACEAE } \\
\hline Maytenus aquifolia Mart. & espinheira-santa & at & 108,130 \\
\hline \multicolumn{4}{|l|}{ COMBRETACEAE } \\
\hline Terminalia brasiliensis (Camb.) Eichler & amarelinho & av & $25,28,44$ \\
\hline \multicolumn{4}{|l|}{ EBENACEAE } \\
\hline Diospyrus inconstans Jacq. & fruta-de-jacu & av & 65,128 \\
\hline \multicolumn{4}{|l|}{ ERYTHROXYLACEAE } \\
\hline Erythroxylum deciduum A.St.-Hil. & cabelo-de-negro & av & 70 \\
\hline Erythroxylum pelleterianum A.St.-Hil. & & at & 280 \\
\hline
\end{tabular}


continuação

\begin{tabular}{|c|c|c|c|}
\hline Família/Espécie & Nome popular & $\mathrm{P}$ & $\mathrm{N}^{\circ}$ de coleta \\
\hline \multicolumn{4}{|l|}{ EUPHORBIACEAE } \\
\hline Actinostemon communis (Müll.Arg.) Pax & laranjeira-brava & $\mathrm{ab}$ & $2,41,124$ \\
\hline Actinostemon concolor (Spreng.) Müll.Arg. & laranjeira-do-mato & $\mathrm{ab}$ & 275,284 \\
\hline Croton floribundus (L.) Spreng. & capixingui & av & 78,116 \\
\hline Pera glabrata (Schott) Baill. & sapateiro & av & 43,283 \\
\hline Sapium glandulatum ( Vell.) Pax & leiteiro & av & 258 \\
\hline \multicolumn{4}{|l|}{ FLACOURTIACEAE } \\
\hline Casearia sylvestris $\mathrm{Sw}$. & café-do-mato & av & $3,4,208$ \\
\hline \multicolumn{4}{|l|}{ LACISTEMATACEAE } \\
\hline Lacistema hasslerianum Chodat & cafeeiro-do-mato & at & 52,270 \\
\hline \multicolumn{4}{|l|}{ LAURACEAE } \\
\hline Endlicheria paniculata (Spreng.) J.F.Macbr. & canela-frade & av & 153,234 \\
\hline Nectandra grandiflora Nees & canela-amarela & av & 251 \\
\hline Ocotea catharinensis $\mathrm{Mez}$ & canelinha-preta & & 101,195 \\
\hline Ocotea diospyrifolia (Meissn.) Mez & canela & av & 57,267 \\
\hline Ocotea lanata (Nees) Mez & canela-lanosa & at & 144 \\
\hline Ocotea odorifera (Vell.) Rohwer & canela-sassafrás & av & 53 \\
\hline Ocotea puberula (Rich.) Nees & canela-parda & av & 247 \\
\hline \multicolumn{4}{|l|}{ LEGUMINOSAE } \\
\hline \multicolumn{4}{|l|}{ Caesalpinioideae } \\
\hline Bauhinia forficata Link & unha-de-vaca & av & 9,93 \\
\hline Bauhinia longifolia (Bong.) Steud. & unha-de-vaca & av & 46,193 \\
\hline Cassia ferruginea (Schrad.) Schrad. ex DC. & chuva-de-ouro & av & 273 \\
\hline Copaifera langsdorffii Desf. & pau-d'óleo & av & 35,194 \\
\hline Hymenaea courbaril $\mathrm{L}$. & jatobá & av & 136,189 \\
\hline Peltophorum dubium (Spreng.) Taub. & farinha-seca & av & 245 \\
\hline Schizolobium parahyba (Vell.) S.F.Blake & guapuruvu & av & 230 \\
\hline Senna cernua (Balb.) H.S. Irwin \& Barneby & & $a b$ & \\
\hline Senna multijuga (Rich.) H.S.Irwin \& Barneby & canafístula & av & 170 \\
\hline \multicolumn{4}{|l|}{ Mimosoideae } \\
\hline Acacia farnesiana (L.) Willd. & & $\mathrm{ab}$ & 181 \\
\hline Acacia polyphylla DC. & monjoleiro & av & 119,175 \\
\hline Acacia recurva Benth. & & $a b$ & 150 \\
\hline Calliandra foliolosa Benth & caliandra & $a b$ & 40 \\
\hline Inga vera (DC.) T.D.Penn. & ingá & av & 39,152 \\
\hline Piptadenia gonoacantha (Mart.) MacBr. & pau-jacaré & av & 22,107 \\
\hline Pithecellobium incuriale Benth. & angico-branco & av & 23 \\
\hline \multicolumn{4}{|l|}{ Papilionoideae } \\
\hline Andira fraxinifolia Benth. & angelim-rosa & av & 27,141 \\
\hline Centrolobium tomentosum Guill. ex Benth. & araribá & av & 36,129 \\
\hline Dalbergia brasiliensis Vogel & caviúna-preta & at & 115 \\
\hline Dalbergia frutescens (Vell.) Britton & rabo-de-bugio & av & 159 \\
\hline Dalbergia villosa Benth. & & av & 42 \\
\hline Lonchocarpus campestris Mart. ex Benth. & sapuva & av & 158 \\
\hline Lonchocarpus cultratus (Vell.) H.C.Lima & embira-de sapo & av & 92,276 \\
\hline Lonchocarpus mиehlbergianus Hassl. & manga-brava & av & 168,200 \\
\hline Luetzelburgia auriculata (Fr.All.) Ducke & guaiçara & av & 165 \\
\hline Machaerium hirtum (Vell.) Stellfeld & bico-de-pato & av & 137 \\
\hline Machaerium nictitans (Vell.) Benth. & jacarandá-ferro & av & 188,255 \\
\hline Machaerium scleroxylon Tul. & caviúna & av & 133 \\
\hline Machaerium stipitatum (DC.) Vogel & jacarandá-roxo & av & 192 \\
\hline Myroxylon peruiferum L.f. & cabreúva & av & 241,246 \\
\hline Ormosia arborea (Vell.) Harms & olho-de-cabra & av & 26 \\
\hline
\end{tabular}


continuação

\begin{tabular}{|c|c|c|c|}
\hline Família/Espécie & Nome popular & $\mathrm{P}$ & $\mathrm{N}^{\circ}$ de coleta \\
\hline \multicolumn{4}{|l|}{ MALVACEAE } \\
\hline Abutilon peltatum K.Schum. & & $\mathrm{ab}$ & 242,243 \\
\hline \multicolumn{4}{|l|}{ MELASTOMATACEAE } \\
\hline Miconia cinnamomifolia (DC.) Naudin & jacatirão & av & 184 \\
\hline Miconia discolor A.DC. & & at & 244 \\
\hline Miconia hymenonervia (Raddi) Cogn. & & av & 226 \\
\hline Miconia langsdorffii Cogn. & & $\mathrm{ab}$ & 80,191 \\
\hline \multicolumn{4}{|l|}{ MELIACEAE } \\
\hline Cabralea canjerana (Vell.) Mart. & canjerana & av & 163 \\
\hline Cedrela fissilis Vell. & cedro-rosa & av & 34,105 \\
\hline Guarea guidonia (L.) Sleumer & cedro-branco & at & 202,220 \\
\hline Trichilia catigua A.Juss. & pau-de-espeto & at & 142 \\
\hline Trichilia elegans A.Juss. & pau-de-ervilha & $\mathrm{ab}$ & 97,162 \\
\hline Trichilia pallida $\mathrm{Sw}$. & catiguá-comum & av & 120,213 \\
\hline \multicolumn{4}{|l|}{ MONIMIACEAE } \\
\hline Mollinedia widgrenii A.DC. & pimenteira-brava & at & 265 \\
\hline Siparuna guianensis Aubl. & limão-bravo & at & 104 \\
\hline \multicolumn{4}{|l|}{ MORACEAE } \\
\hline Ficus citrifolia $\mathrm{P}$. Miller & figueira-mata-pau & av & 210 \\
\hline Ficus enormis (Mart. ex Miq.) Miq. & figueira-branca & av & 212 \\
\hline Ficus guaranitica Chodat ex Chodat \& Vischer & figueira-branca & av & 118 \\
\hline Maclura tinctoria (L.) D.Don. ex. Steud. & taiúva & av & 77,139 \\
\hline \multicolumn{4}{|l|}{ MYRSINACEAE } \\
\hline Myrsine coriacea (Sw.) R.Br. ex Roem. \& Schult. & capororoca & av & 233 \\
\hline Myrsine umbellata Mart. & capororoca & av & 271 \\
\hline Myrsine umbrosa Mart. Ex Miq. & capororoca & av & 228 \\
\hline \multicolumn{4}{|l|}{ MYRTACEAE } \\
\hline Calycorectes acutatus (Miq.) Toledo & amarelinho & av & 37,285 \\
\hline Calyptranthes clusiifolia (Miq.) O.Berg. & jaborandi & av & 204 \\
\hline Campomanesia guazumaefolia (Camb.) O.Berg. & sete-capotes & at & 289 \\
\hline Eugenia excelsa O.Berg. & & av & 282 \\
\hline Eugenia glazioviana Kiaersk. & guamirim & av & 127,256 \\
\hline Eugenia moraviana Berg. & cambuim & at & 261 \\
\hline Eugenia sp. & & at & 64 \\
\hline Gomidesia affinis (Cambess.) D.Legrand & brasa-viva & av & 145,196 \\
\hline Myrcia rostrata DC. & cambuí & av & 75,272 \\
\hline Myrciaria ciliolata Camb. & cambuí, Cereja & at & 262 \\
\hline Myrciaria tenella (DC.) O.Berg. & cambuí & at & 102,207 \\
\hline \multicolumn{4}{|l|}{ NYCTAGINACEAE } \\
\hline Guapira opposita (Vell.) Reitz & maria-mole & av & 32,121 \\
\hline \multicolumn{4}{|l|}{ PHYTOLACCACEAE } \\
\hline Seguieria langsdorffii Moq. & agulheiro & av & $50,54,85$ \\
\hline \multicolumn{4}{|l|}{ PIPERACEAE } \\
\hline Piper amalago L. & & $\mathrm{ab}$ & 156 \\
\hline Piper claussenianum (Miq.) C.DC. & & $\mathrm{ab}$ & 176,236 \\
\hline Piper crassinervium H.B.K. & & $\mathrm{ab}$ & 171,238 \\
\hline Piper glabratum Kunth & & $\mathrm{ab}$ & 172 \\
\hline Piper hostmannianum (Miq.) C.DC. & & $\mathrm{ab}$ & 237 \\
\hline Pothomorphe umbellata (L.) Miq. & & $\mathrm{ab}$ & 232 \\
\hline \multicolumn{4}{|l|}{ POLYGONACEAE } \\
\hline Coccoloba cf. glaziovii Lind. & & av & 197 \\
\hline
\end{tabular}


continuação

\begin{tabular}{|c|c|c|c|}
\hline Família/Espécie & Nome popular & $\mathrm{P}$ & $\mathrm{N}^{\circ}$ de coleta \\
\hline \multicolumn{4}{|l|}{ PROTEACEAE } \\
\hline Roupala brasiliensis Klotszch & carne-de-vaca & av & 67,217 \\
\hline \multicolumn{4}{|l|}{ ROSACEAE } \\
\hline Prunus myrtifolia (L.) Urb. & coração-de-negro & av & 229,266 \\
\hline \multicolumn{4}{|l|}{ RUBIACEAE } \\
\hline Alibertia concolor (Cham.) Schum. & & at & 187,205 \\
\hline Alseis floribunda Schott & tarumã & av & 154 \\
\hline Chomelia nitida (H.B.K.) DC. & jasmim-do-mato & $\mathrm{ab}$ & 148,257 \\
\hline Chomelia sericea Müll.Arg. & & $\mathrm{ab}$ & 61,131 \\
\hline Coutarea hexandra (Jacq.) K.Schum. & murta & av & 155 \\
\hline Psychotria vellosiana Benth. & orelha-de-gato & at & 811,287 \\
\hline Simira sampaioana (Standl.) Steyerm. & guatambu-preto & av & 223,288 \\
\hline \multicolumn{4}{|l|}{ RUTACEAE } \\
\hline Esenbeckia grandiflora Mart. & guaxupita & at & 1 \\
\hline Metrodorea nigra A.St.-Hil. & carrapateira & at & 60,281 \\
\hline Zanthoxylum caribaeum Lam. & & av & 198 \\
\hline Zanthoxylum hiemalis A.St.-Hil. & mamica-de-porca & av & 201 \\
\hline Zanthoxylum monogynum A.St.-Hil. & & av & 219 \\
\hline \multicolumn{4}{|l|}{ SAPINDACEAE } \\
\hline Cupania vernalis Camb. & arco-de-peneira & av & 173 \\
\hline Matayba juglandifolia (Camb.) Radlk. & pau-de-pombo & av & 254 \\
\hline \multicolumn{4}{|l|}{ SAPOTACEAE } \\
\hline Chrysophyllum gonocarpum (Mart. \& Eichl.) Engl. & guatambu-de-sapo & av & 29 \\
\hline \multicolumn{4}{|l|}{ SOLANACEAE } \\
\hline Brunfelsia uniflora (Pohl) D.Don & manacá-de-cheiro & $\mathrm{ab}$ & 62 \\
\hline Cyphomandra fragrans Sendtn. & & $\mathrm{ab}$ & 179 \\
\hline Solanum bistellatum L.B. Sm. \& Downs & & $\mathrm{ab}$ & 277 \\
\hline Solanum concinum Schott ex Sendtn. & & $a b$ & 240 \\
\hline Solanum granulosoleproso Dunal & jurubeba & av & 88,178 \\
\hline Solanum variabile Mart. & joveva & $\mathrm{ab}$ & 177 \\
\hline \multicolumn{4}{|l|}{ STERCULIACEAE } \\
\hline Helicteres ovata Lam. & saca-rolha & art & 157,218 \\
\hline \multicolumn{4}{|l|}{ STYRACACEAE } \\
\hline Styrax camporum Pohl & canelão & at & 122,123 \\
\hline Styrax pohlii A.DC. & benjoeiro & at & 74 \\
\hline \multicolumn{4}{|l|}{ TILIACEAE } \\
\hline Luehea divaricata Mart. & açoita-cavalo & av & 138,222 \\
\hline \multicolumn{4}{|l|}{ ULMACEAE } \\
\hline Trema micrantha (L.) Blume & pau-pólvora & av & 174 \\
\hline \multicolumn{4}{|l|}{ URTICACEAE } \\
\hline Urera baccifera (L.) Gaudich. & urtigão & art & 164 \\
\hline \multicolumn{4}{|l|}{ VERBENACEAE } \\
\hline Aegiphila sellowiana Cham. & & av & 134,231 \\
\hline Aloysia virgata (Ruiz \& Pav.) Juss. & lixa, lixeira & at & 63,260 \\
\hline Vitex polygama Cham. & maria-preta & av & 72,143 \\
\hline \multicolumn{4}{|l|}{ VIOLACEAE } \\
\hline Hybanthus atropurpureus (A.St.-Hil.) Taub. & erva-de-veado & $a b$ & 190 \\
\hline \multicolumn{4}{|l|}{ VOCHYSIACEAE } \\
\hline Qualea jundiahy Warm. & jundiaí & av & 94 \\
\hline Vochysia tucanorum Mart. & pau-de-tucano & av & 99,146 \\
\hline
\end{tabular}


específica tiveram valores de constância relativa de $60 \%$ ou mais (tabela 3): Leguminosae, Meliaceae, Euphorbiaceae, Myrtaceae, Rutaceae, Rubiaceae e Lauraceae, tendo Leguminosae e Meliaceae 100\% de constância relativa.

Os gêneros de maior constância relativa e maior riqueza específica nos diferentes trechos da FESM no estado de São Paulo (tabela 3) foram: Ocotea, Miconia, Solanum, Eugenia, Machaerium, Myrcia e Zanthoxylum. Os gêneros de maior constância relativa e de maior riqueza específica nos levantamentos da FESS no estado de São Paulo (tabela 3) foram: Machaerium, Aspidosperma, Trichilia, Eugenia, Zanthoxylum e Solanum.

Com base no resultado do teste G, Meliaceae e Rutaceae apresentaram constância relativa

Tabela 3. Constância relativa das famílias e gêneros de maior riqueza específica nos diferentes trechos da Floresta Estacional Semidecídua Submontana (FESS) ou Montana (FESM) no estado de São Paulo. Resultados do teste G $\left(\alpha=0,05, \mathrm{G} . \mathrm{L} .=1\right.$ e $\left.\mathrm{G}_{\mathrm{c}}=3,841\right)$ maiores que o crítico $\left(\mathrm{G}_{\mathrm{c}}\right)$ indicam diferença significativa entre a FESS e a FESM.

Table 3. Relative constancy of the richest genera and families in diferent surveys of the Montane (FESM) and Submontane (FESS) Semideciduous Seasonal Forest in São Paulo State. G test $(\alpha=0.05$, d.f. $=1)$ results greater than the critical value $\left(\mathrm{G}_{\mathrm{c}}=3.841\right)$ indicate significant difference between FESS and FESM.

\begin{tabular}{lccc}
\hline Gêneros ou Famílias & \multicolumn{2}{c}{ Constância relativa $(\%)$} & Teste G \\
\cline { 2 - 3 } & FESS & FESM & \\
\hline Machaerium & 93,3 & 62,5 & 4,60 \\
Aspidosperma & 86,6 & 18,7 & 15,72 \\
Trichilia & 86,6 & 25,0 & 12,88 \\
Eugenia & 75,0 & 62,5 & 1,16 \\
Zanthoxylum & 60,0 & 37,5 & 1,59 \\
Solanum & 53,3 & 68,7 & 0,80 \\
Ocotea & 40,0 & 87,5 & 16,16 \\
Miconia & 26,6 & 68,5 & 5,68 \\
Myrcia & 13,3 & 50,0 & 5,04 \\
Leguminosae & 100,0 & 93,7 & 1,34 \\
Meliaceae & 100,0 & 43,7 & 15,40 \\
Euphorbiaceae & 93,3 & 100,0 & 1,48 \\
Myrtaceae & 93,3 & 93,7 & 0,0 \\
Rutaceae & 93,3 & 25,0 & 16,82 \\
Rubiaceae & 73,3 & 87,5 & 1,02 \\
Lauraceae & 60,0 & 87,5 & 3,16 \\
Solanaceae & 26,6 & 75,0 & 7,54 \\
Asteraceae & 13,3 & 56,2 & 6,62 \\
Melastomataceae & 0,0 & 50,0 & 13,22 \\
\hline
\end{tabular}

significativamente maior nas FESS que na FESM; Solanaceae, Asteraceae e Melastomataceae, maior constância relativa nas FESM que nas FESS (tabela 3). Ocotea, Miconia e Myrcia apresentaram constância relativa significativamente maior nas FESM que nas FESS, enquanto Machaerium, Aspidosperma e Trichilia apresentaram constância significativamente maior nas FESS que nas FESM (tabela 3).

A mata da Fazenda Bela Vista apresentou algumas espécies não citadas ou muito pouco citadas para a Floresta Estacional Semidecídua no estado de São Paulo: Acacia recurva, A. farnesiana, Alseis floribunda, Aspidosperma camporum, Bactris setosa, Coccoloba glaziovii, Erythroxylum pelleterianum, Eupatorium inulaefolium, Lonchocarpus campestris, Luetzelburgia auriculata, Piper claussenianum, P. glabratum, P. hostmannianum, Senna cernua, Simira sampaioana, Solanum bistellatum, Tabebuia ochracea, T. serratifolia.

\section{Discussão}

A pequena proporção de espécies de arbustos e arvoretas encontradas na mata da Fazenda Bela Vista está de acordo com a observação de Rodrigues et al. (1989), segundo a qual a FESM apresenta os estratos arbustivo e herbáceo pouco desenvolvidos, quando comparados com os de florestas em altitudes mais baixas. Também é possível que o limite mínimo de $3 \mathrm{~cm}$ de DAP adotado no levantamento da floresta da Fazenda Bela Vista tenha excluído a maioria dos arbustos. Mas, observações no campo mostraram que o hábito arbustivo é pouco freqüente na floresta da Fazenda Bela Vista. No entanto, a pequena ocorrência de arbustos também pode ser decorrente das perturbações sofridas pela mata estudada.

A concentração de riqueza específica em poucas famílias tem sido observada por outros autores em outros locais de várias formações florestais no estado de São Paulo e no Brasil, com cerca de $20 \%$ do total de famílias representando cerca de metade das espécies amostradas (Martins 1991, Meireles 2004). As dez famílias de maior riqueza específica na floresta da Fazenda Bela Vista, com alguma variação, também se apresentaram como as mais ricas em outros levantamentos de espécies lenhosas realizados na Floresta Estacional do estado de São Paulo (Leitão Filho 1982, Santos \& Kinoshita 2003). A concentração da riqueza específica em Leguminosae também foi observada na grande maioria das florestas não só do interior paulista como também de todo o Brasil 
(Leitão Filho 1987, Martins 1991, Santos 2003). Famílias como Leguminosae, Euphorbiaceae, Myrtaceae, Rubiaceae e Lauraceae estão entre as mais ricas em espécies lenhosas no mundo (Takhtajan 1997, Cronquist 1981) e estão entre as famílias com alta constância e alta riqueza tanto na formação Montana quanto na Submontana da Floresta Estacional Semidecídua (Leitão Filho 1982, Santos \& Kinoshita 2003). Na flora mundial, Leguminosae representa cerca de 8,3\%, Euphorbiaceae 4,39\%, Rubiaceae 3,80\%, Myrtaceae ao redor de $1,75 \%$ e Lauraceae 1,17\% do total de espécies Magnoliopsida (Cronquist 1981), sendo especialmente ricas em espécies na região tropical (Good 1974). Se uma família é muito rica em espécies e tem uma distribuição muito ampla, espera-se que, em qualquer local da área de sua distribuição, ela conste dentre as famílias de maior constância e maior riqueza. Portanto, esperava-se que essas famílias fossem das mais ricas também na mata da Fazenda Bela Vista.

Os resultados da comparação feita entre as FESS e as FESM indicaram que as formações Montana e Submontana da Floresta Estacional Semidecídua apresentam diferenças na composição de famílias. Torres et al. (1997) não encontraram diferenças entre as formações Submontana e Montana no estado de São Paulo, mas consideraram a presença ou a ausência de todas as famílias, enquanto este presente estudo considerou apenas as famílias de maior constância e maior riqueza específica em cada uma dessas formações.

Piper, que apresentou cinco espécies na mata da Fazenda Bela Vista, foi citado como um dos gêneros mais ricos apenas nos levantamentos que incluíram espécimes de pequeno diâmetro. Portanto, como a variação do tamanho do menor indivíduo incluído nos diferentes levantamentos parece influenciar muito na riqueza de Piper, não há como discutir se esse gênero é característico de alguma formação da Floresta Estacional Semidecídua no estado de São Paulo. Ocotea, Miconia e Myrcia tiveram riqueza e constância significativamente maiores na formação Montana que na Submontana. Na mata da Fazenda Bela Vista, Myrcia apresentou apenas uma espécie, enquanto Ocotea e Miconia apresentaram alta riqueza de espécies. Por outro lado, Aspidosperma e Trichilia apresentaram riqueza específica e constância significativamente maiores na formação Submontana que na Montana. Como esperado, na mata da Fazenda Bela Vista, uma formação Montana, Aspidosperma apresentou apenas uma espécie. No entanto, Trichilia apresentou três espécies na mata em estudo. Na mata da Fazenda Bela Vista, Tabebuia, Cordia, Dalbergia, Lonchocarpus, Acacia, e Myrsine apresentaram riqueza específica relativamente alta. Em alguns trechos da formação Montana paulista, esses gêneros apresentaram riqueza específica alta, mas, no geral, mostraram baixa constância relativa. Embora Meira Neto et al. (1989), Leitão Filho (1992), Salis et al. (1995) e Torres et al. (1997) tivessem observado uma mudança na composição de espécies entre florestas em altitudes menores ou maiores que $700 \mathrm{~m}$, não analisaram diferenças em nível de gênero.

Quase metade das espécies arbustivas e arbóreas apontadas como indicadoras de floresta de altitude por Meira Neto et al. (1989) também foi encontrada na mata da Fazenda Bela Vista. Lá ocorreram tanto táxons considerados exclusivos de grandes altitudes e ou climas frios e úmidos quanto táxons considerados típicos de baixas altitudes e ou climas secos e quentes, segundo a classificação proposta por Salis et al. (1995). A mistura de táxons considerados característicos de uma ou outra condição também foi encontrada quando consideramos os níveis de família ou gênero. Concluímos que, na Floresta Estacional Semidecídua no estado de São Paulo, a formação Montana se distingue da Submontana, tendo algumas famílias (Solanaceae, Asteraceae e Melastomataceae) e gêneros (Ocotea, Miconia e Myrcia) com maior constância e maior riqueza específica na formação Montana. Por outro lado, na formação Submontana outras famílias (Meliaceae e Rutaceae) e gêneros (Machaerium, Aspidosperma e Trichilia) ocorrem com maior constância e maior número de espécies.

As várias espécies pouco citadas ou não citadas para a Floresta Estacional Semidecídua do estado de São Paulo, encontradas na mata da fazenda Bela Vista, reforçam a idéia da distribuição geográfica restrita da maioria das espécies arbóreas sugerida por Torres et al. (1997). Com o padrão predominante de distribuição geográfica restrita da maioria das espécies arbóreas, resulta ser muito importante não só preservar e proteger os fragmentos florestais que ainda restam no interior do estado de São Paulo, como também conhecer sua flora.

Agradecimentos - Ao Senhor José A. Cremasco pela permissão da realização do presente estudo em sua Fazenda Bela Vista. Aos pesquisadores taxonomistas do Departamento de Botânica da Unicamp: Dra. Ana Maria Goulart de Azevedo Tozzi, Dra. Angela Borges Martins, Dr. João Semir, Jorge Yoshio Tamashiro e Dr. Washington Marcondes-Ferreira Neto; do Departamento de Botânica da USP: Dra. Cintia Kameyama, Dr. José Rubens Pirani e Dr. Paulo Sano; do Instituto de Botânica do Estado de São Paulo: Dra. Maria 
Lucia Kawasaki; do Instituto Florestal do Estado de São Paulo: Dr. João Batista Baitello e Osni Aguiar; do Herbário do IAC: Dra. Sigrid L. Jung-Mendaçolli; do Curso de Pós-Graduação em Biologia Vegetal da Unicamp: Andreia Barbosa, Angela L.Bagnatori Sartori, Kazue Matsumoto, Maria de Fátima Freitas e Vidal de Freitas Mansano, pelo auxílio na identificação do material botânico. A Yukio Makino e Rodrigo B. Singer, pelo auxílio no trabalho de campo. À Capes, pela bolsa de mestrado concedida à primeira autora.

\section{Referências bibliográficas}

BAITELLO, J.B. \& AGUIAR, A.T. 1982. Flora arbórea da serra da Cantareira (São Paulo). Silvicultura em São Paulo 16A:582-590.

BAITELLO, J.B., AGUIAR, O.T., ROCHA, F.T., PASTORE J.A. \& ESTEVES, R. 1992. Florística e fitossociologia do estrato arbóreo de um trecho da Serra da Cantareira (Núcleo Pinheirinho) - SP. Revista do Instituto Florestal 4:291-297.

BERNACCI, L.C. \& LEITÃO FILHO, H.F. 1996. Flora fanerogâmica da floresta da Fazenda São Vicente, Campinas, SP. Revista Brasileira de Botânica 19:149-164.

BERTONI, J.E.A. 1984. Composição florística e estrutura fitossociológica de uma floresta do interior do estado de São Paulo: Reserva Estadual de Porto Ferreira. Dissertação de Mestrado, Universidade Estadual de Campinas, Campinas.

BERTONI, J.E.A., MARTINS, F.R., MORAES, J.L. \& SHEPHERD, G.J. 1988. Composição florística e estrutura fitossociológica do Parque Estadual de Vaçununga, Santa Rita do Passa Quatro, SP - Gleba Praxedes. Boletim Técnico do Instituto Florestal 42:149-170.

BRUMMITT, R.B. \& POWELL, C.E. 1992. Authors of plant names. Royal Botanic Gardens, Kew.

CARDOSO-LEITE, E. 1995. Ecologia de um fragmento florestal em São Roque, SP: florística, fitossociologia e silvigênese. Dissertação de Mestrado, Universidade Estadual de Campinas, Campinas.

CATHARINO, E.L.M. 1989. Estudos fisionômico-florístico e fitossociológico em matas residuais secundárias no município de Piracicaba, SP. Dissertação de Mestrado, Universidade Estadual de Campinas, Campinas.

CAVASSAN, O., CESAR, O. \& MARTINS, F.R. 1984. Fitossociologia da vegetação arbórea da Reserva Estadual de Bauru, estado de São Paulo. Revista Brasileira de Botânica 7:91-106.

CESAR, O. \& LEITÃO FILHO, H.F. 1990. Estudo florístico quantitativo de mata mesófila na Fazenda Barreiro Rico, município de Anhembi, SP. Revista Brasileira de Biologia 50:133-147.

COSTA, L.G.S. \& MANTOVANI, W. 1995. Flora arbustivoarbórea de trecho de mata mesófila, na Estação Ecológica de Ibicatu, Piracicaba (SP). Hoehnea 22:47-59.
CRONQUIST, A. 1981. An integrated system of classification of flowering plants. Columbia University Press, New York.

CRONQUIST, A. 1988. The evolution and classification of flowering plants. Botanical Garden, New York.

GABRIEL, J.L.C. \& PAGANO, S.N. 1993. Composição florística do estrato arbóreo de floresta mesófila semidecídua de encosta, no município de Botucatu, SP. Arquivos de Biologia e Tecnologia 36:185-206.

GANDOLFI, S., LEITÃO FILHO, H.F. \& BEZERRA, C.L.F. 1995. Levantamento florístico e caráter sucessional das espécies arbustivo-arbóreas de uma floresta mesófila semidecídua no município de Guarulhos, SP. Revista Brasileira de Biologia 55:753-767.

GAUCH, H.G. 1982. Multivariate analysis in community ecology. Cambridge University Press, Cambridge.

GOOD, K. 1974. The geography of the flowering plants. Longman, London.

KOTCHETKOFF-HENRIQUES, O. \& JOLY, C.A. 1994. Estudo florístico e fitossociológico em uma mata mesófila da Serra do Itaqueri, Itirapina, estado de São Paulo, Brasil. Revista Brasileira de Biologia 54:477-487.

KUHLMANN, M. 1942. Observações gerais e contribuições ao estudo da flora e fitofisionomia do Brasil: estudos florísticos e fitofisionômicos realizados na região de Monte Alegre, município de Amparo, São Paulo, em maio de 1942. Instituto de Botânica, São Paulo.

KUHLMANN, M. \& KÜHN, E. 1947. A flora do Distrito de Ibiti (ex Monte Alegre), município de Amparo. Instituto de Botânica, São Paulo.

LEITÃO FILHO, H.F. 1982. Aspectos taxonômicos das florestas do estado de São Paulo. Silvicultura em São Paulo 16A:197-206.

LEITÃO FILHO, H.F. 1987. Considerações sobre a florística de florestas tropicais e subtropicais do Brasil. Boletim do Instituto de Pesquisas e Estudos Florestais 35:41-46.

LEITÃO FILHO, H.F. 1992. A flora arbórea da Serra do Japi. In História natural da Serra do Japi: Ecologia e preservação de uma área florestal no Sudeste de Brasil. (L.P.C. Morellato org.). Editora da Unicamp, Campinas, p. 40-62.

MARTINS, F.R. 1991. Estrutura de uma floresta mesófila. Editora da Unicamp, Campinas.

MATTHES, L.A.F. 1980. Composição florística, estrutura e fenologia de uma floresta residual do Planalto Paulista: Bosque dos Jequitibás (Campinas, SP.). Dissertação de Mestrado, Universidade Estadual de Campinas, Campinas.

MATTOS, J.R. \& MATTOS, N.F. 1982. Contribuição ao conhecimento da flora do Parque Estadual de Campos de Jordão, SP. Silvicultura em São Paulo 16A:647-662.

MEIRA NETO, J.A.A., BERNACCI, L.C., GROMBONE, M.T., TAMASHIRO, J.Y. \& LEITÃO FILHO, H.F. 1989. Composição florística da floresta semidecídua de altitude do Parque Municipal da Grota Funda (Atibaia, estado de São Paulo). Acta Botanica Brasilica 3:51-74. 
MEIRELES, L.D. 2004. Composição florística do estrato arbóreo de uma floresta de altitude da Serra da Mantiqueira, Monte Verde, MG. Dissertação de Mestrado, Universidade Estadual de Campinas, Campinas.

MÜELLER-DOMBOIS, H. \& ELLENBERG, D. 1974. Aims and methods of vegetation ecology. Wiley, New York.

NICOLINI-GABRIEL, E.M. \& PAGANO, S.N. 1992. Composição florística do estrato arbóreo de floresta mesófila semidecídua no município de Jahu, SP. Arquivos de Biologia e Tecnologia 35:725-748.

OLIVEIRA, J.B., CAMARGO, M.N., ROSSI, M. \& CALDERANO-FILHO, B. 1999. Mapa pedológico do estado de São Paulo. Instituto Agronômico de Campinas, Campinas.

OLIVEIRA FILHO, A.T., SCOLFORO, J.R.S. \& MELLO, M. 1994. Composição florística e estrutura comunitária de um remanescente de floresta semidecídua montana em Lavras, MG. Revista Brasileira de Botânica 17:167-182.

PAGANO, S. \& LEITÃO FILHO, H.F. 1987. Composição florística do estrato arbóreo de mata mesófila semidecídua, no município de Rio Claro (estado de São Paulo). Revista Brasileira de Botânica 10:37-47.

PASTORE, J.A., AGUIAR, O.T., ESTEVES, R. \& SILVA, C.A.F. 1992. Flora arbóreo-arbustiva do Parque Chico Mendes, município de São Bernardo do Campo (SP). Revista do Instituto Florestal 4:269-273.

POLHILL, R.M. \& RAVEN, P.H. (eds.). 1981. Advances in Legume Systematics. Part I. Royal Botanic Gardens, Kew.

PONÇANO, W.L., CARNEIRO, C.D.R., BISTRICHI, C.A., ALMEIDA, F.F.M., PRANDINI, F.L. 1981. Mapa geomorfológico do estado de São Paulo. v.2. IPT, São Paulo.

ROBIM, M.J., PASTORE, J.A., AGUIAR, O.T. \& BAITELLO, J.B. 1990. Flora arbóreo-arbustiva e herbácea do Parque Estadual de Campo do Jordão (SP). Revista do Instituto Florestal 2:31-53.

RODRIGUES, R.R., MORELLATO, L.P.C., JOLY, C.A. \& LEITÃO FILHO, H.F. 1989. Estudo florístico em um gradiente altitudinal de mata estacional mesófila semidecídua, na serra do Japi, Jundiaí, SP. Revista Brasileira de Botânica 12:71-84.

ROSSI, L. 1994. A flora arbóreo-arbustiva da mata da Reserva da Cidade Universitária "Armando de Salles Oliveira" (São Paulo, Brasil). Boletim do Instituto de Botânica 9:1-105.

ROZZA, A.F. 1997. Florística, fitossociologia e caracterização sucessional em uma floresta estacional semidecidual: Mata da Virgínia, Matão, SP. Dissertação de Mestrado, Universidade Estadual de Campinas, Campinas.
SALIS, S.M., SHEPHERD, G.J. \& JOLY, C.A. 1995. Floristic comparison of mesophytic semideciduous forests of the interior of the state of São Paulo, Southeast Brazil. Vegetatio 119:155-164.

SANTOS, K. 2003. Caracterização florística e estrutural de onze fragmentos de mata Estacional Semidecídua da área de proteção ambiental do município de Campinas - SP. Tese de doutorado, Universidade Estadual de Campinas, Campinas.

SANTOS, K. \& KINOSHITA, L.S. 2003. Flora arbustivoarbórea de fragmento de floresta estacional semidecidual do Ribeirão Cachoeira, município de Campinas - SP. Acta Botanica Brasilica 17:325-486.

SILVA, A.F. 1989. Composição florística e estrutura fitossociológica do estrato arbóreo da Reserva Florestal Prof. Augusto Ruschi, São José dos Campos - SP. Tese de Doutorado, Universidade Estadual de Campinas, Campinas.

SOKAL, R.R. \& ROHLF, F.J. 1995. Biometry. W.H. Freeman, New York.

STRANGHETTI, V. \& RANGA, N.T. 1998. Levantamento florístico das espécies vasculares da floresta estacional semidecidual da Estação Ecológica Paulo de Faria - SP. Revista Brasileira de Botânica 21:289-298.

TAKHTAJAN, A. 1997. Diversity and classification of flowering plants. Columbia University Press, New York.

TOLEDO FILHO, D.V., LEITÃO FILHO, H.F., BERTONI, J.E.A., BATISTA, E.A. \& PARENTE, P.R. 1993. Composição florística do estrato arbóreo da Reserva Estadual de Águas da Prata - (SP). Revista do Instituto Florestal 5:113-122.

TORRES, R.B. 1989. Estudos florísticos em mata secundária na Estação Ecológica de Angatuba, Angatuba (São Paulo). Dissertação de Mestrado, Universidade Estadual de Campinas, Campinas.

TORRES, R.B., MARTINS, F.R. \& GOUVEIA, L.S.K. 1997. Climate, soil, and tree flora relationship in forests in the state of São Paulo, southeastern Brasil. Revista Brasileira de Botânica 20:41-49.

VELOSO, H.P., RANGELFILHO,A.L.R. \& LIMA, J.C.A. 1991. A classificação da vegetação brasileira adaptada a um sistema universal. IBGE, Rio de Janeiro.

VIEIRA, M.GL., MORAES, J.L., BERTONI, J.E.A., MARTINS, F.R. \& ZANDARIN, M.A. 1989. Composição florística e estrutura fitossociológica da vegetação arbórea do Parque Estadual de Vaçununga, Santa Rita do Passa Quatro (SP). Gleba Capetinga Oeste. Revista do Instituto Florestal 1:135-159.

WALTER, H. 1972. Ecology of tropical and subtropical vegetation. Oliver \& Boyd, London. 